\title{
An HACCP approach integrating quantitative microbial risk assessment and shelf life prediction
}

\author{
V. Popov, D. P. Janevska \& R. Gospavic \\ Wessex Institute of Technology, Ashurst Lodge, Southampton, UK
}

\begin{abstract}
The presence and growth of specific food pathogens (SFP) and specific spoilage organisms (SSO) in food products is associated, respectively, with an increase in probability for illness in consumers and decreased shelf life of product. Technology, which would assist the food supply chain operators with accurate and timely estimations of the risk levels to consumers and controlled shelf life in the food supply chain, would represent a valuable tool for increasing the safety and quality of the food product. In this work, a quantitative microbial risk assessment (QMRA) and shelf life prediction (SLP) models and computer codes have been developed to assist the hazard analysis and critical control point (HACCP) procedures. Although the SLP is not usually part of the HACCP, it has been added to the system in order to increase the efficiency of the complete operation including food quality and safety. The QMRA and SLP modules can operate as an integral part of the decision support system (DSS) but also could be used as standalone software tools for prediction and analysis in food supply chains. The communication between QMRA and SLP and DSS includes the input parameters from DSS related to temperature profile for a specific supply chain, food type, kind of microbial organisms and lab results which are provided at different points in the supply chain. The QMRA and SLP use their own internal data bases for model parameters for different supply chains and food products. The data bases could be updated or extended if necessary. The available data cover fish and poultry supply chains but QMRA and SLP modules are developed as general tools and could be used for different supply chains and different kind of food.
\end{abstract}

Keywords: HACCP, quantitative microbial risk assessment, shelf life prediction. 


\section{Introduction}

The presence of specific food pathogens (SFP) in food products will not cause illness in people unless the number of cells is high enough. The SFP becomes dangerous when the number of cells increases sufficiently to cause illness or produce sufficient amount of toxins that are harmful. Since the probability for pathogen entry in the food supply chain cannot be zero, in order to prevent illness in consumers due to consumption of the product, conditions which can lead to increase in microbial counts need to be kept under control. The Hazard Analysis and Critical Control Point (HACCP) concept helps to achieve such control of the conditions in the supply chain, if properly designed and implemented. As HACCP is a qualitative approach the Quantitative Microbial Risk Assessment (QMRA) is required in order to conduct the hazard analysis step for the pathogen microorganisms and to identify required control mechanisms for the critical control points. In this work it is analysed how HACCP can benefit from QMRA module and also SLP which would increase the food quality and reduce the waste.

\section{HACCP and current regulations}

Today HACCP is an internationally recognized system (set of guidelines) and is widely used for safe food production. If properly applied it could help to achieve a higher level of food safety. For the system to work it needs to be applied by food companies along the food chain.

The food law in EU, Regulation 178/2002 that applies from 1st January 2005 states that food companies have primary responsibility for safe food. From 1st January 2006 new food hygiene law Regulation 852/2004 requires all food companies in all member states (except farms) to operate food safety programmes based on the seven HACCP principles contained in the Codex Alimentarius.

It is expected that the future HACCP implementations will incorporate the Quantitative Microbial Risk Assessment (QMRA). HACCP is a qualitative approach and it has been pointed out that qualitative risk assessment cannot address the process's inherent variability in any meaningful manner [1]. Since QMRA is a quantitative approach, a synthesis of both approaches should produce a food safety system which will be able to offer higher reliability. One of the aspects where QMRA can clearly help HACCP is that it can provide quantitative estimates of the risk to consumers taking into account the variability of the parameters in the food supply chain. It is also possible that HACCP integrated with QMRA will be easier to compare for equivalence world-wide, due to the added quantitative dimension. If the QMRA is implemented in practice, the determination of equivalence between very different systems should be more straightforward. 


\section{QMRA and SLP implementation}

The QMRA consists of the following elements (see Figure 1): (i) hazard identification, (ii) exposure assessment, (iii) hazard characterization, (iv) risk characterization.

\subsection{Hazard identification}

Hazard identification is the first step in the QMRA where the specific foodborne pathogens (SFP) relevant to the considered supply chain are identified. This step is supply chain specific, in terms of SFP that might be present or introduced at a certain point in the supply chain, and is carried out by screening.

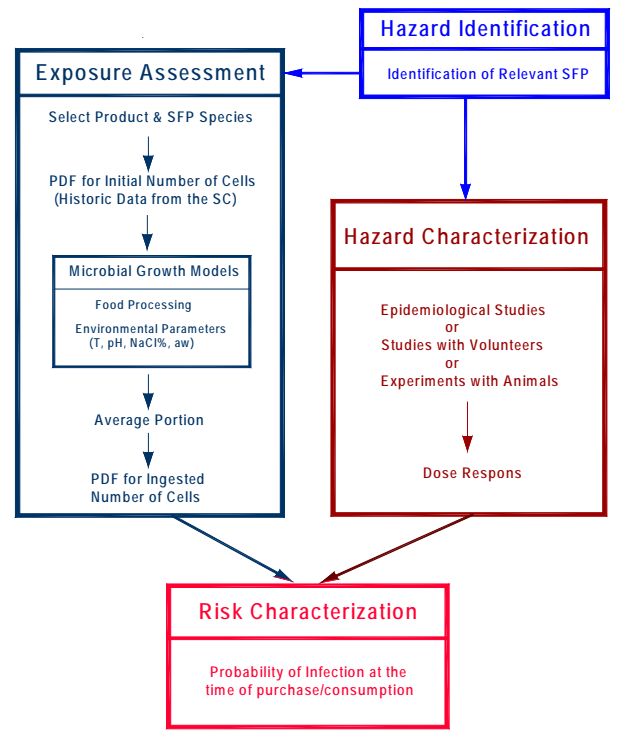

Figure 1: $\quad$ Elements of the QMRA.

\subsection{Exposure assessment}

The main purpose of exposure assessment is to establish the probability for the ingested number of cells of SFP by the consumer. The uncertainty in different environmental and supply chain parameters can be included into the mathematical model by using stochastic models, e.g., Monte Carlo simulation.

In order to establish the probability density function for the ingested number of SFP, quantitative information on the incidence, prevalence and count of pathogens in the product are required. This information has to be collected through testing of the product over certain period of time, sufficiently long in duration to produce reliable data. 


\subsubsection{Microbial growth models}

The microbial growth models are used to predict the growth of microorganisms in the product under specified environmental conditions. During the development of the mathematical models for growth of particular microorganism the first step is to perform laboratory experiments in order to provide the necessary data. The second step is to prepare a supply chain specific microbial growth model. It is important to note that such models would be reliable as long as the conditions in the supply chain stay within the range of parameters used in the experiments.

\subsubsection{Primary models}

The primary models provide information on the growth of certain bacteria in constant environmental conditions [2, 3] and they are used in both, the QMRA and the SLP. Since the temperature varies in supply chains the primary microbial growth models cannot cover the whole supply chain.

\subsubsection{Secondary models}

The secondary models $[4,5]$ are able to predict bacterial growth in a condition of variable environmental parameters, e.g., T, $\mathrm{pH}, \mathrm{NaCl} \%$, water activity $\left(a_{\mathrm{w}}\right)$, modified atmosphere. The most important parameter in the secondary models is usually the temperature.

\subsubsection{Deterministic and stochastic models}

The microbial growth models can be deterministic or probabilistic/stochastic. The deterministic models do not take into account random distributions of the initial cell population as well as random behaviour of the bacterial organisms during the multiplication. On the other hand the stochastic models can take into account the high level of variability and uncertainty in environmental and biological parameters and as outcome can produce the probability for microbial concentration at different time instants or at different points within the supply chain. It is convenient to use the probability density function for defining the number of SFP cells as an input parameter for the stochastic microbial growth models.

\subsection{Hazard characterization}

An essential part of the QMRA is a suitable dose response model for estimating the probability for illness due to ingestion of certain number of SFP cells. However, the accurate dose-response relation is difficult to describe for two reasons: (1) the variability in both host susceptibility and microorganism infectivity; and (2) the lack of experimental data [6, 7].

Several statistical models have been used to describe microbial dose response relation. The most often reported dose response models for SFP in the literature are the log-normal, log-logistic, simple exponential, flexible exponential, BetaPoisson, Weibull-Gamma and Gompertz [8, 9]. 


\subsection{Implementation of the SLP}

The SLP model uses a microbial growth model similarly to the QMRA. The SSO selected is product and supply chain specific. The rejection point, in terms of microbial count, is determined by experiments involving sensory evaluation.

\section{Near-real-time predictions of the QMRA and SLP}

By implementing the QMRA and SLP modules in a supply chain a near-realtime data processing and provision of information to the supply chain operators on the risk levels to consumers and remaining shelf-life of the product taking into account variations in environmental parameters, can be obtained. Such implementation requires technology which can transfer data automatically from sensors in the supply chain to the decision support system (DSS), which integrates the QMRA and SLP modules. The frequency with which the data is transmitted to the DSS depends on the environmental parameters of interest and the SC.

Figure 2 shows a possible implementation of the QMRA module. The necessary Real-Time-Data (RTD) and input parameters for risk assessment analysis of microbial contamination along the supply chain is provided through communication with the DSS and from the database. The QMRA itself is imagined as a generic software module which can operate for various food supply chains, providing that the database with the necessary information exists. As can be seen in Figure 2 a possibility for update of the databases is added. Such updates are desirable in order to take into account possible changes in the supply chain. The experiments for establishing the parameters of the microbial growth models are usually conducted in a laboratory environment. In the experiments if a product from the supply chain is used higher probability for agreement between the model predictions and actual microbial growth rates in the supply chain is expected. Fine tuning of the models can be further carried out in the specific supply chain in order to achieve higher accuracy of the predictions. The above discussion is also valid for the SLP model.

In the implementation described in Figure 2, an efficient supply of real time data along the supply chain is essential for successful operation of the DSS. A simplified data transmission between the DSS and QMRA/SLP is presented in Figure 3.

It may occur that in some parts of the food supply chain such real time data supply is not possible, and for these parts the data should be stored and sent to the DSS as soon as the possibility arises. An alternative to the system presented in Figure 3 is presented in Figure 4. This implementation differs mainly by the strategy by which the SC data is delivered to the DSS. While the DSS in Figure 3 operates with real time data delivered via mobile management units (MMU) or stationary management units (SMU), the data in this case is delivered via uploading data from sensors, e.g. temperature loggers, placed in boxes with the product, into the system at the (critical) control points (CCPs) in order to perform the analysis of the remaining shelf life and to estimate the probability 
for illness in consumers. It must be emphasized that though in Figure 4 the data is transferred at CCP, it is not necessary to transfer the data at CCP only as there may be few in the SC, the data can be transferred at newly established Control Points (CPs) in the SC which would be used specifically for this purpose. Therefore, the update of the system database in this case is not continual, realtime update, but it is at predetermined points in the supply chain. This is a much cheaper version of the system, which should suit smaller SCs and their available budget.

The SLP can be implemented as deterministic or stochastic model while the QMRA should be implemented as a stochastic model since it will provide a probability for illness upon consumption of the product. The deterministic model uses less number of operations and therefore is faster; however the utility of the stochastic model is higher.

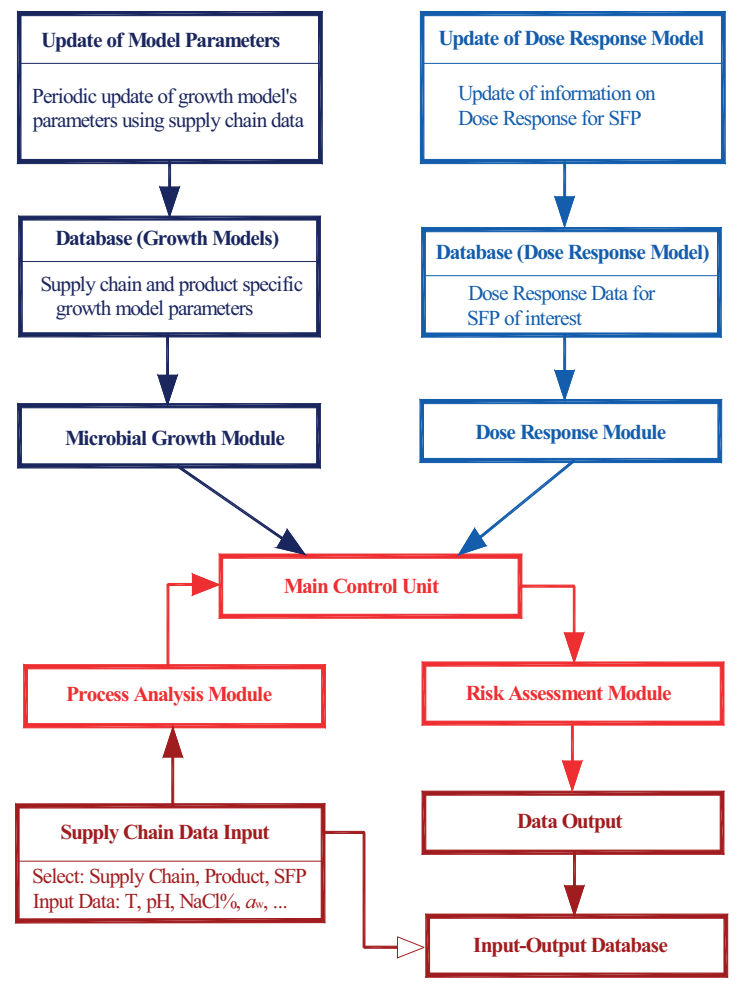

Figure 2: Block diagram of the QMRA module (adapted from Janevska et al. [10]). 


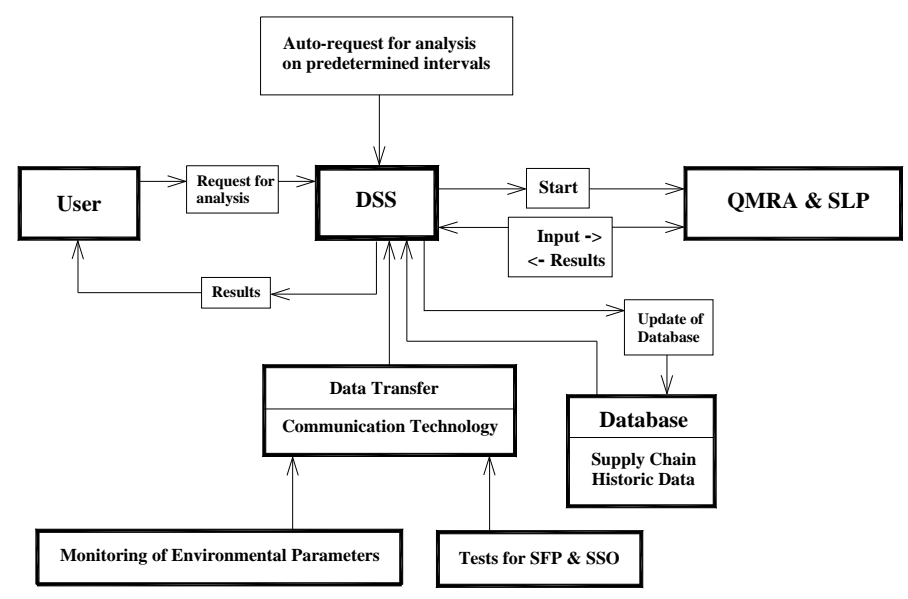

Figure 3: $\quad$ Block diagram of interaction of DSS and QMRA and data transfer.

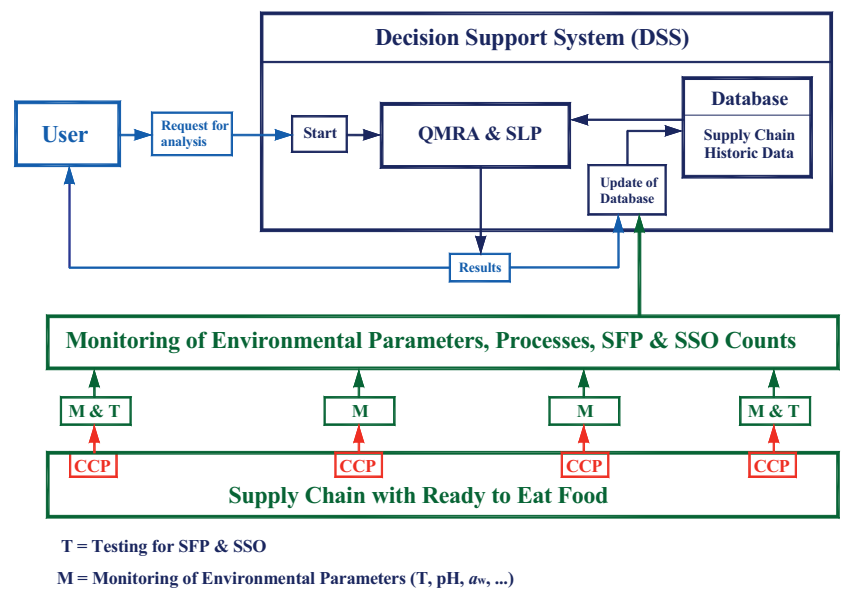

Figure 4: Implementation of QMRA and SLP within DSS without the possibility of real-time data supply.

\section{Benefits of integrating HACCP with QMRA and SLP}

The integration of the QMRA and SLP within the DSS with the HACCP would bring the following improvements in the SC: (i) The risk assessment provided by QMRA will help the HACCP decisions by providing quantitative information; (ii) More accurate and nearly-continuous SC predictions on the risk levels for the consumers and remaining shelf life depending on the monitoring data in the SC; (iii) Warnings sent to SC operators independent of the HACCP and location of 
control points in the SC; (iv) The verification procedures of the HACCP for pathogen bacteria is helped by the QMRA; (v) Procedures (testing) implemented in HACCP to verify accuracy of the QMRA and SLP; (vi) The corrective actions for the HACCP can be added to the DSS, therefore reducing possibility for mistakes; (vii) Full compatibility of the QMRA-HACCP system with the HACCP procedures can be achieved; (viii) Early detection of change in the SC which require corrective actions by periodical analysis of historic data using the QMRA and SLP models.

\section{Adaptation of the HACCP-QMRA system to changes in the SC}

The QMRA and the SLP are estimating the Risk Level (RL) and the remaining Shelf Life (SL) for the product and this enables one to analyse the trends in RL and SL in order to establish whether there are significant changes in these two SC parameters. Such changes in RL and SL due to any change in internal properties of the food product or changes in the external environmental parameters in the SC could be periodical/seasonal or they could be long term changes. The benefits of applying the HACCP-QMRA/SLP approach to estimating RL and SL in SCs stems from the fact that the HACCP alone does not enable the SC operators to quantify the impact of the slight changes in parameters, e.g. temperature, in the product on the RL to consumers and SL of the product. Since the HACCP requires continuous/periodic verification, QMRA can provide a very efficient tool for this task. Instead of looking just at environmental parameters, e.g. temperature, in the SC, the QMRA can provide estimates of changes in RL due to change in the temperature profile of the SC. Statistical methods should be employed in analysis of RL and SL over a period of time in order to establish whether a significant change in the SC has occurred which requires attention. The initial implementation of the QMRA and SLP would be based on laboratory microbial growth experiments, however, after certain period of time when there is enough amount of SC data, calibration of the models could be carried out which would bring the predictions closer to the actual SC state.

Such implementation of the QMRA and SLP would be able to adapt its accuracy despite the changes in the SC, regardless of the type of changes, which might affect the RL or SLP.

Once implemented, the HACCP, the QMRA and the SLP would be considered as a single system which would secure the required quality and safety of the product.

The HACCP can be periodically verified using the QMRA. The QMRA can provide estimate when the RL to consumer has reached unacceptable levels and can trigger review of the HACCP procedures in order to bring the risk to an acceptable level. The QMRA can include statistical analysis which would report a trend of increase in levels of risk, though the critical limits in the control points have not been exceeded. This would allow time for corrective actions before the HACCP and the SC safety are compromised. During the redesign of the HACCP 
the QMRA can be used to define the necessary changes in order to achieve the risk reduction. Processes and procedures in the SC can be reviewed and adapted in this way in order to reduce the risk to consumers and increase the SL.

The HACCP would have to be adapted in a way to include tests which would collect sufficient data for calibration of the models. It should also include checks for predictions given by the QMRA and can also be used as a platform that would include steps for use of the SLP. Though the SLP is not part of the HACCP it makes sense to use the HACCP in order to implement the SLP in an efficient way without adding a separate system to the SC.

\section{Conclusions}

The HACCP-QMRA/SLP approach which has been developed offers a number of advantages in respect to the usual HACCP stand-alone system. The practical aspects of the implementation of such system have been elaborated.

The new system utilizes a novel HACCP-QMRA approach and a possible integration of the Shelf Life Prediction (SLP) model as well, to secure safety and quality of food products. Various aspects of the implementation are discussed together with the benefits of such system for increasing the safety and quality of food products in supply chains. It is expected that the system will be able to provide early warning for changes in risk levels to consumers due to consumption of the product as well as shelf life of the product in the supply chain.

\section{Acknowledgement}

The financial support by the European Commission through the FP6 - Food Quality and Safety programme, Contract No. FOOD-CT-2004-513953, is gratefully acknowledged.

\section{References}

[1] Buchanan, R. L. and Whiting, R. C. (1998). Risk assessment: a means for linking HACCP plans and public health. J. Food Protect., 61(11), 15311534.

[2] Baranyi J. and Roberts T. A. (2006) Mathematics of predictive food microbiology, International Journal of Food Microbiology, 26, 199-218.

[3] Swinnen, I. A. M., Bernaerts, K., Dens, E. J. J., Geeraerd, A. H. and Van Impe, J. F. (2004) Predictive modelling of the microbial lag phase: a review, International Journal of Food Microbiology, 94, 137-159.

[4] Baranyi J. and Roberts T. A. (1994) A dynamic approach to predicting bacterial growth in food - Review Paper. International Journal of Food Microbiology, 23, 277-294.

[5] Gospavic R., Kreyenschmidt J., Bruckner S., Popov V. and Haque N. (2008) Mathematical modelling for predicting the growth of Pseudomonas 
spp. in poultry under variable temperature conditions. International Journal of Food Microbiology, 127, 290-297.

[6] Farber, J. M., Rose, W. H. and Harwig, J. (1996) Health risk assessment of Listeria monocytogenes in Canada, Intl. J. Food Microbiol., 30, 145-154.

[7] Holcomb, D. L., Smith, M. A., Ware, G. O., Hung, Y. C., Brackett, R. E. and Doyle, M. P. (1999) Comparison of six dose-response models for use with foodborne pathogen, Risk Anal. 19(6), 1091-1100.

[8] Coleman, M., Marks, H. (1998) Topics in dose-response modelling. J. of Food Protection, 61(11), 1550-1559.

[9] Medema, G. J., Teunis, P. F. M., Havelaar, A. H. and Haas, C. N. (1996) Assessment of the dose-response relationship of Campylobacter jejuni, Intl. J. Food Microbiol., 30, 101-111.

[10] Janevska, D. P., Gospavic, R., Pacholewicz, E. and Popov, V. (2010) Application of a HACCP-QMRA approach for managing the impact of climate change on food quality and safety. Food Research International, 43/7, 1915-1924. 